\title{
Probes: paths to photostability
}

\author{
Vivien Marx
}

\begin{abstract}
Blinking and photobleaching of fluorophores cause challenges in a whole range of imaging experiments. Here are some ways researchers are approaching fluorophore photostability.
\end{abstract}

When amateur or professional astronomers see twinkling stars, they know atmospheric turbulence is playing tricks on their eyes. But in the research laboratory, it is not a trick when fluorophores blink, dim or photobleach.

Blinking can create artifacts, says Scott Blanchard, a biophysicist at Weill Cornell Medical College. It can mean scientists miss the cellular event they want to observe or risk misinterpreting a biological process. Blinking can get in the way of observing and measuring single-molecule trajectories over time, says Luke Lavis, a researcher at the Howard Hughes Medical Institute's Janelia Research Campus.

Quantum dots are quite photostable fluorophores, which is why they are great for television sets, says Lavis. But quantum dots blink plenty, are large and are hard to conjugate to other molecules. To photostabilize other fluorophores that are smaller and more common in biomedical labs, such as genetically encoded fluorescent proteins (FPs) and small-molecule dyes, Lavis, Blanchard and others apply a variety of strategies ${ }^{1-3}$.

\section{Fluorescent proteins}

Genetically encoded FPs blink and photobleach to varying degrees, although this behavior is not always problematic. "For most fluorescence imaging, you are looking at vast numbers of molecules at once, so the blinking just averages out and is not relevant to the experiment," says Robert Campbell, a chemist and FP developer at the University of Alberta.

Photobleaching can, however, ruin longterm fluorescence applications, he says, and blinking is a challenge for single-molecule imaging. These issues may explain why the number one request Campbell hears from other scientists is "Please improve fluorescent

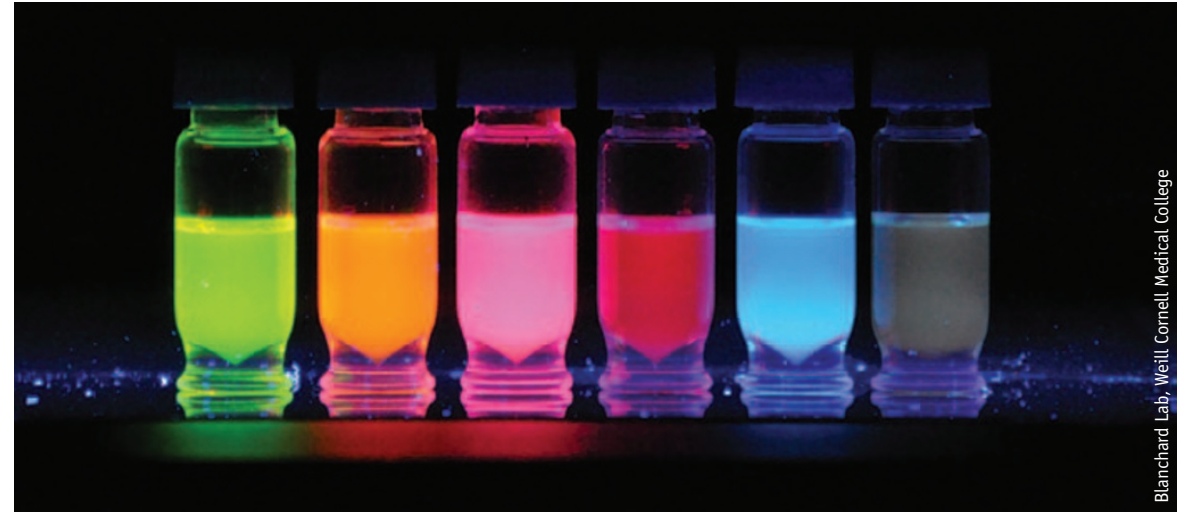

Fluorophores across the visible spectrum can be engineered to be ever more photostable.

protein photostability!" His sense is that the community is near the limits of what is possible to accomplish with the current FP templates.

He and his team worked to improve the photostability of a cyan FP known as monomeric teal fluorescent protein (mTFP). And although mTFP was quite photostable, they were disappointed to find it was roughly as photostable as enhanced green fluorescent protein (EGFP). "Despite our best efforts," he says, "we were unable to surpass the performance of one of the original, and still one of the best, fluorescent proteins."

Labs seeking extreme photostability might turn to alternative FP scaffolds based on a protein that reversibly binds to a fluorogenic organic chromophore, says Campbell. With this approach, they can avoid the kind of 'lights out' response inherent to the chemistry of GFP. GFP's chromophore is generated from its own amino acids and remains covalently bound. A single photobleaching event renders the entire protein useless. "In contrast, if the protein could spit out the bleached chromophore and bind to a fresh one, the apparent rate of photobleaching would dramatically decrease," says Campbell. Some potential candidates for this strategy are phytochrome-derived proteins with biliverdin chromophores and the eel-derived UnaG protein with a bilirubin chromophore. But Campbell does not think that these alternative scaffolds will ever replace FPs as the choice for genetically encoded probes for all imaging experiments. Yet, he says "they could certainly find a niche in applications where photostability is paramount."

The elegance and convenience of having a cell make its own fluorophore outweighs all other considerations for all but a very small number of potential applications, says Campbell. "If I wanted to perform long-term tracking of a cell surface receptor, I would use a quantum dot," he says. "Other than that, FPs all the way!"

FPs, says Lavis, "are great because the cell is the chemist-it makes the fluorophore for you and puts it where you want." But FPs are not as bright as organic dyes, which, in his view, limits their utility in single-molecule tracking. Organic dyes, on the other hand, are small and can easily be hitched onto biomolecules. Scientists are not bound, Lavis 

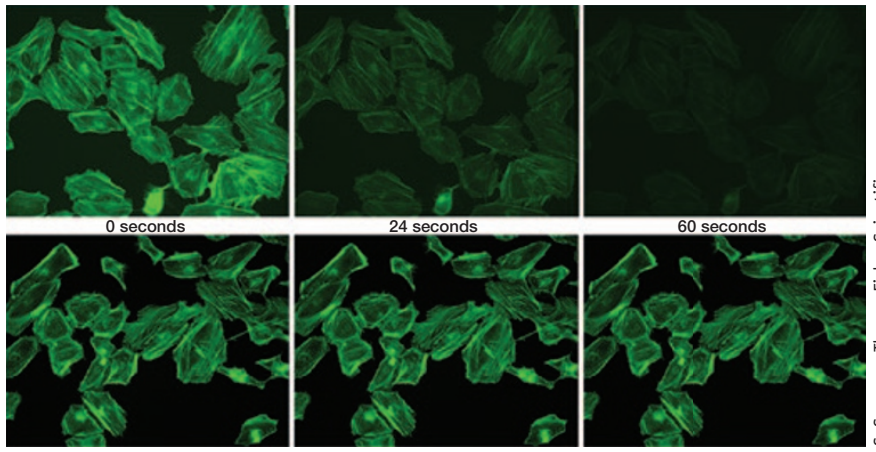

Solution additives can make a difference. Here, fixed HeLa cells mounted in phosphate-buffered saline without (top) or with (bottom) the additive SlowFade Diamond.

jests, to the 'tyranny of the ribosome', and instead "we can use the entire chemical lexicon to make the dyes."

\section{Photostable dyes}

Dye-making is ancient, as is much of the chemistry used, says Lavis. In 2011, he and his team found an easier way to make rhodamine dyes, which chemists previously synthesized with a method developed in the 1880s.

Lavis kept thinking about the dimethyl amino groups in many fluorophores, which shift the emitted light to red wavelengths but also hamper photostability. In new research, he replaced these dimethyl amino groups with fourmembered azetidines, creating socalled Janelia Fluor (JF) dyes" . "This is a minor change in structure but causes a large increase in brightness and photostability," he says. One of the dyes, JF549, is twice as bright and lasts twice as long as the classic tetramethylrhodamine.

Everything spoke against trying the azetidine system, says Lavis. Colleagues, even chemists, were doubtful and gave renditions of "I'm not sure this is going to work, Luke; that ring looks really strained." Only after two years did he convince himself to try the experiment, emboldened by molecular modeling results indicating that the steps would make the dyes brighter and more photostable. The prediction held true.

Lavis sends out the new dyes to other labs to try and also shares them with colleagues at Janelia Research Campus (Box 1). The dyes are easy to use, he says, and labs can also make them on their own. Undergraduates with two weeks of organic synthetic chemistry under their belts can make large quantities of these dyes without a hitch.

In testing, Lavis has found his JF dyes to be photostable in live-cell experiments and useful for in vitro single-molecule experiments. He can also make the dyes blink in a controlled fashion for super-resolution microscopy experiments.

Mark Bates, a postdoctoral fellow at the Max Planck Institute for Biophysical Chemistry, also makes use of controlled fluorophore blinking for super-resolution imaging. Through repeated cycles of illumination, during which fluorophores are activated and then switched off, fluorophore positions can be determined. Bates applied this process to co-develop stochastic optical reconstruction microscopy (STORM) with his PhD advisor, Harvard University researcher Xiaowei Zhuang.

But, says Bates, when a pulse of activation light is sent to a switchable fluorophore, the chance of turning it on is not $100 \%$. And the same is true when switching it off. Even in the most controlled experiment, it is always a random process. "When we want the fluorophore to be on, we want it to stay on," says

\section{BOX 1 SHARING AND DISTRIBUTING}

Logistical issues arise once a lab has developed a new probe and colleagues want to test it. Labs distribute probes on their own, work with companies or combine both approaches.

Luke Lavis is in talks with nearly a dozen companies to license his new azetidinyl dyes for different applications. Because negotiations are ongoing, he does not wish to disclose the company names. What industry likes, he says, is both the small size and the easy syntheses.

Until a commercial source is found and the details worked out, the lab freely distributes the dyes to the community from the Janelia Research Campus. He is glad to see other labs beginning to work with them for single-molecule imaging. Colleagues at Janelia imaged and characterized the dynamics of some transcription factors and enhancer systems, in this case the Sox2 enhancer, in live embryonic stem cells ${ }^{8}$. They used the dyes JF549 and JF646 in live-cell imaging to localize, track and map Sox2 binding events and applied lattice light-sheet-based singlemolecule imaging.

Scott Blanchard has launched a company called Lumidyne Technologies to commercialize his organic fluorophores, and the start-up has licensed some of them from Weill Cornell Medical College. In the past, he has shared fluorophores under development in his lab freely with others and continues to do. He has hired scientists to manage the process as volume has increased. Feedback from colleagues will show how the dyes perform in a variety of contexts and systems. When it comes time to scale up production, he says, the chemistry is often better handled in an industrial setting.

At Thermo Fisher Scientific, Kamran Jamil and Michael O'Grady also mine user experiences with antifade agents and dyes. They collect positive experiences and do not ignore the gripes, says O'Grady. The company also partners with labs to beta-test new reagents.

Many scientists approach the company excited about their new dyes and reagents, says Hsiaoli Chen, who handles in-licensing and partnerships at Thermo Fisher. To evaluate dye submissions internally, she says, the team looks for robustness, photostability and great quantum yield. Frequently, however scientists submit their dyes too early, before they have validated biological data to share, including tests in live cells or cell culture. She and her team look for effects of any photostabilizing additive or dye on cell viability, proliferation and other cellular functions because future customers will be asking them for these data.

Often a university will prefer to file a patent before sharing data, a practice Chen understands. The more data that can be shared, the easier the company's evaluation of a potential dye to in-license becomes. She and her team might in-license a compound from one university and in-license a synthesis approach from another. In-house chemists and biologists also want to see whether the dyes have broad applicability and how the dyes or reagents stack up against others. "Ideally," she says, "it is not just incremental improvement." 


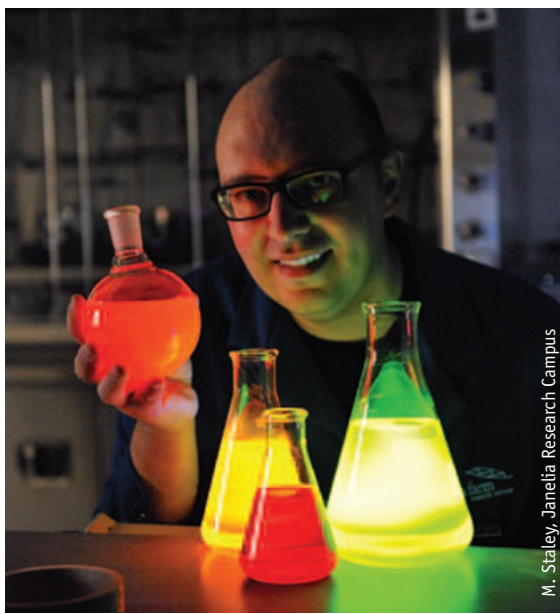

Organic dyes are brighter than fluorescent proteins, says Luke Lavis.

Bates. And when it should be off, it should stay that way. "We like control." But in practice, a molecule is not utterly controllable: many influences lead it to change states.

For STORM, ideally he would like more stable fluorescence on and off states as well as tight control over the transitions between those states. Were he doing different experiments, such as watching single-molecule dynamics by monitoring the efficiency of fluorescence resonance energy transfer (FRET) between two dyes, he says he would want the dyes to maintain "a perfectly stable brightness, without blinking, and without photobleaching."

\section{A blink of an eye}

Actually, all fluorophores blink constantly, says Bates. They cycle through excited and dark states to deliver a train of photons that serves as the signal for a detector or camera. Plenty can derail this photon train, leading to photolability. "A fluorophore is a small, fragile molecule in a big world," says Bates.

The molecule can twist, as in the case of cis-trans isomerization of the red fluorescent dye Cy5. The cis state is nonfluorescent, whereas the trans state fluoresces. There are also electronic interactions that can cause a fluorophore to go dark. When a fluorophore absorbs a photon, one of its ground-state electrons is promoted to a higher energy level. In that state, the electron might interact with a neighboring molecule in such a way that its spin flips. The electron is then trapped in what is called the triplet state, and the fluorophore remains dark, says Bates.

Alternatively, a fluorophore might collide with other molecules, or there might be FRET to a nearby fluorophore. In such cases the fluorophore's energy is not emitted as light but is dissipated as heat or energy that is transferred to another molecule.

The excited state of a fluorophore is also highly reactive. As Blanchard says, the fluorophores run the risk of damaging themselves when they are in triplet or redox states. When a fluorophore interacts with molecular oxygen, it generates singlet oxygen and also oxygen radicals. Photostability takes a hit when the fluorophore reacts with what can be a little army of damaging reactive oxygen species, says Blanchard. Photostabilizing efforts address this highly reactive excited state.

To stabilize fluorophores such as cyanines, rhodamines and oxazines, scientists can use what are called reducing and oxidizing systems, in which reducing and oxidizing agents are balanced ${ }^{5}$.

Oxygen scavengers help to capture reactive oxygen species before they can lead a fluorophore down a path to photobleaching. Some additives that do so are Trolox; cyclooctatetraene (COT); 4-nitrobenzyl alcohol (NBA); $p$-phenylenediamine (PPD), which is used in the commercial product Vectashield made by Vector Labs; and 4-diazabicyclo[2.2.2] octane (DABCO), which is used in the product Ibidi-MM. These chemicals work well for fixed cells but can be toxic to live cells.

\section{Stability in live cells}

Some photostabilizing additives can be used with live cells, such as the enzymatic compound OxyFluor, made by Oxyrase. This enzyme formulation is reported to reduce photobleaching by decreasing oxygen radical formation.

Thermo Fisher Scientific is about to launch a photostabilizing additive for live-cell imaging. The company already sells oxygen

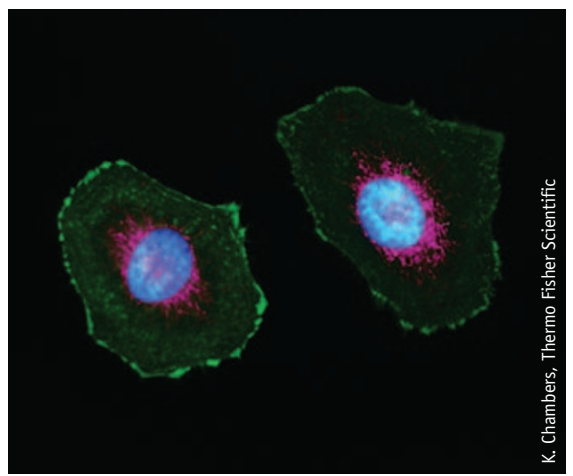

The solution additive ProLong Live can be added to cell cultures, here HeLa cells. According to the vendor, the additive can help keep various fluorophores photostable for around three times as long as with medium alone. scavenger antifade agents such as SlowFade and ProLong. ProLong cures cells into a gel and is intended for use on samples that will be stored for an extended period, whereas SlowFade does not cure cells.

Last year, the company launched new versions of these reagents, called ProLong Diamond and SlowFade Diamond, which were developed in-house to offer enhanced photostability, says Kamran Jamil, who manages these products. The company sought additives to address the increased use of FPs for imaging at far-red wavelengths and the multiplexing of fluorophores, he says.

Scientists can fix cells at a particular phase in the cell cycle, put the cells on slides and keep them for years, says Michael O'Grady, who manages Thermo Fisher Scientific's R\&D for fluorescence applications and reagents. Given that an increasing number of labs do live-cell imaging, the company developed ProLong Live, the photostabilizing additive for live-cell applications that is about to be introduced. Although the company does not wish to disclose details, it does say that this new reagent is in the class of oxygen scavengers.

Photostability is not just about additives but also about the imaging process. The company's technical-hotline staff helps researchers less familiar with fluorescence microscopy. Jamil and his team have discovered that too many researchers expose fluorophores to more light than needed, which exacerbates photostability issues.

\section{Beyond the classic way}

$\beta$-mercaptoethanol (BME) is a classic solution additive to stabilize fluorophores. BME was the fluorophore stabilizer handed to Blanchard during his graduate training. But $\mathrm{BME}$ is also toxic to some in vitro systems and to live cells, a fact that kept him from studying the molecular machine of his interest, the ribosome. "That's where my search, my personal journey for understanding fluorophore photophysics, began," he says.

Additives can enhance photostability, but not in every experimental setup. Usually labs have no choice but to try them to find out what might work, says Blanchard. These substances tend to have been developed for fixed cells, in which toxicity does not matter.

A number of new types of additives work through triplet-state quenching. A team at McGill University recently used $\mathrm{Ni}^{2+}$ as a solution additive and triplet quencher to photostabilize the organic dye Cy3 (ref. 6).

Some experiments just cannot work with 
traditional additives, says Blanchard. Many additives can interfere with membraneprotein function. Blanchard's workaround is to quench fluorophores intramolecularly. He and his team used these fluorophores in recently published work on the aspartate transporter ${ }^{7}$. He photostabilizes fluorophores by conjugating triplet-stage quenchers to dyes. The idea is to shorten fluorophore triplet-state lifetime, reduce the number of reactive oxygen species and have fewer unwanted reactions that lead to blinking and photobleaching.

By following this strategy, Blanchard chemically modified cyanine fluorophores such as Cy5 by linking them to COT, NBA and Trolox, solution additives also used for photostability. This approach made Cy5, a dye dogged by blinking and photobleaching issues, an order of magnitude more photostable. It can be used with other fluorophores, and for live-cell imaging. He hopes the strategy has opened the door to a more defined and quantitative way of talking about how to improve fluorophore performance.

\section{Ways forward}

Many fluorophores, including ones from commercial and academic sources and that have long been in use, are just not perfect, says Blanchard, nor are they strong enough for single-molecule and live-cell imaging. "We are exiting the era of fixedcell imaging," he says. "And we're moving into the era of wanting, desiring to track the behaviors of single molecules."

Fluorophores are getting better, but tracking single molecules calls for dyes with 100- to 1,000-fold performance enhancement, he says. Brightness is certainly king, but this brightness must also be long and stable. And researchers need a way to conjugate a fluorophore specifically to the molecular machine of interest so that they know what the experiment is reporting on.

Blanchard believes that probing fluorophore enhancement with a greater understanding of mechanisms allows researchers to engineer greater fluorophore photostability and to bring out the natural abilities of these molecules.

Better mechanistic understanding also helps scientists to explore how the performance of fluorophores can be influenced by attachment to the protein or nucleic acid target of interest. This can lead to more efficient conjugation to the target than is currently often the case. "You don't want your fluorophore reacting with all sorts of things," says Blanchard. "When it reacts, you want to say 'that's my protein." Organic fluorophores, unlike FPs, are not genetically encodable, but Blanchard hopes that approaches to conjugate them to a target specifically and efficiently can make up for that.

Improvements in photostability are likely to go hand in hand with improvements in delivery in order to maximize the potential of fluorescence experiments using organic dyes within living cells.

For all their advantages, many chemical dyes have some downsides that make them not quite ready-made for live-cell experiments, says Lavis. Using dyes in live-cell studies often requires harsh protocols such as microinjection or bead loading to get the compounds or their conjugates into cells.

HaloTags, Snap-tags and other related fusion-protein strategies are letting scientists combine the advantages of genetically encoded FPs with the favorable physics of organic dyes, says Lavis. With these tags, a genetically encoded protein domain can react covalently and specifically with, for example, fluorophores.

Although increased brightness and photostability are important, Bates has high hopes for exploring how best to target organic dyes to a structure of interest. "That's the big challenge in most superresolution projects that I work on," he says.

In Blanchard's view, his lab and others have likely not yet found the optimal way to enhance fluorophore photostability. "My intuition says this is the tip of the iceberg," he says. A deeper understanding of how to engineer better fluorophore performance will herald new fluorophores and will allow researchers to define the rules for better performance predictions.

1. Zheng, Q. et al. Chem. Soc. Rev. 43, 1044-1056 (2014).

2. Yang, S.K., Shi, X., Park, S., Ha, T. \& Zimmerman, S.C. Nat. Chem. 5, 692-697 (2013).

3. Altman, R.B. et al. Nat. Methods 9, 68-71 (2012).

4. Grimm, J.B. et al. Nat. Methods 12, 244-250 (2015).

5. Vogelsang, J. et al. Angew. Chem. Int. Ed. Engl. 47, 5465-5469 (2008).

6. Glembockyte, V., Lincoln, R. \& Cosa, G. J. Am. Chem. Soc. 137, 1116-1122 (2015).

7. Akyuz, N. et al. Nature 518, 68-73 (2015).

8. Liu, Z. et al. elife 3, 04236 (2014).

\section{Vivien Marx is technology editor for Nature and Nature Methods \\ (v.marx@us.nature.com).}

